\title{
Development of a Model for Locating Hubs in a Competitive Environment under Uncertainty: A Robust Optimization Approach
}

\author{
F. Nourzadeh ${ }^{\mathrm{a}}$, S. Ebrahimnejad*b, K. Khalili-Damghania, A. Hafezalkotoba \\ a Department of Industrial Engineering, South Tehran Branch, Islamic Azad University, Tehran, Iran \\ ${ }^{\boldsymbol{b}}$ Department of Industrial Engineering, Karaj Branch, Islamic Azad University, Karaj, Iran
}

\section{PAPER INFO}

\section{Paper history:}

Received 25 August 2019

Received in revised form 30 September 2019

Accepted 08 November 2019

\section{Keywords:}

Competitive Location

Multivariate Lagrange Interpolation Function

Location Hub

Robust Optimization

\section{$\begin{array}{lllllllll}A & B & S & T & R & A & C & T\end{array}$}

This article explores the development of previous models to determine hubs in a competitive environment. In this paper, by comparing parameters of the ticket price, travel time and the service quality of hub airports, airline hubs are divided into six categories. The degree of importance of travel time and travel cost are determined by a multivariate Lagrange interpolation method, which can play an important role in allocating travelers to follower airline hubs. Then, based on the seasonal demand of travelers, we consider travel demand as uncertain parameters. To determine the robust counterpart of this category of hub location models, a robust optimization method is used. Finally, models are tested in a case study. The central results show that the follower airline's income has a considerable growth and can absorb nearly $2 \%$ of travelers of the leader airline due to lower travel costs and travel time compared to that of leader airline.

doi: 10.5829/ije.2020.33.01a.14

\section{INTRODUCTION}

In this paper, to determine the exact location of hubs we develop the model of Marianov et al. [1] by defining a new type of capture sets under uncertainty. We divide the nominated airports for follower airline's hub into six independent categories by comparing parameters of travel cost, travel time and service quality of hubs. By employing multivariate Lagrange interpolation function, we determine the degree of importance of time and cost of travel from the traveler's perspective. The results show that the follower airline's income has a considerable growth compared to the Marianov et al. [1] model and can absorb nearly $2 \%$ of travelers of the leader airline due to lower travel costs and travel time compared to that of leader airline.

Marianov et al. [1] proposed the first hub location problem in a competitive environment. In their model for the hub location of follower player, the leader player has already selected its hub-and-spoke network. The flow from origin to destination passes through only one path (including the maximum of two hubs). They also presented two models for the follower player. In the first model, if the cost of traveler movement by the follower is lower than the leader, then all of the travelers are attracted to leader player hubs. However, in the second model, three capture sets are presented based on the compared cost of travel by the follower and leader player. The members of those sets will attract 50, 75 and 100\% of travelers, respectively.

By changing two models of Marianov et al. [1], Wagner [2] reduced the time needed to achieve an optimal solution for problems with more than 50 spokes and 5 hubs. He developed capture sets, and based on the fact that flow from origin to destination passes through one or two paths, he proposed 6 capture sets. To identify the members of capture sets, Marianov et al. [1] and Wagner [2] just compared the cost of moving traveler by the follower and leader player. However, aside from the movement cost of travelers, there are other parameters such as travel time and service quality of hubs (airports) in the real-world, which can be used as the basis for the player and its under-controlled hubs selection. To overcome the aforementioned problem and determine the members of capture sets more accurately, we compared the travel times in addition to the travel cost of players. Additionally, comparing the service quality of hubs under-controlled of players is the cornerstone of our 
research. Marianov et al. [1] and Wagner [2] used the values of $0.7,0.9$, and 1.1 as well as 50,75 , and $100 \%$ in their capture sets. In this paper, the multinomial Lagrange interpolation method was used to calculate the exact values determined by them. Moreover, the bounds of variables belonged to these sets were determined by considering the degree of importance of parameters of travel time and travel cost from the viewpoint of travelers, the quality of airports and airlines, safety, and delays of airlines.

In the real world, ignoring even one small uncertainty in data will result in an optimal solution that can be insignificant and meaningless. Therefore, various approaches were developed in the literature to gain more realistic optimal solutions. The robust optimization approach is one of the most important approaches in this area. The approaches presented by Soyster [3], Ben-Tal and Nemirovski [4], and Bertsimas and Sim [5] are the main approaches for robust optimization, which are employed in articles related to hub location under uncertainty. Concerning the possibility of controlling the conservativeness of robust solutions and linearity of the method of Bertsimas and Sim [5], was used in this approach to demonstrate the uncertainty in parameters of travel demand to gain more realistic solutions.

To compare the presented model in this paper and the model of Marianov et al. [1], we examined a case study, in which Emirate airline is the leader airline, and follower airline consists of three airlines named Mahan Airline, Iran Air, and Aseman Airline. The spokes were selected from a pool of cities, which passed the largest number of travelers from European countries to East Asian countries by the hubs of Emirate airline. The findings showed that the follower airline's coalition income has a considerable growth compared to the model of Marianov et al. [1].

In the next section, the literature review is presented. In section 3, the model of Marianov et al. [1] is developed. Then, to determine the degree of importance of travel time and cost, we introduced a multivariate Lagrangian interpolation function. In section 4 , the model described in the third section will be presented in uncertainty mode by employing Bertsimas and Sim's approach [5]. In section 5, the model for a real problem is implemented and compared with the model of Marianov et. al [1]. Also, we investigated the impact of change in the problem's parameters on the value of the objective function and presented managerial insights. Concluding remarks is presented in the last section of this paper.

\section{LITERATURE REVIEW}

The most important research related to the subject of this paper is presented here. Sasaki and Fukushima [6] proposed a new model called "Stackelberg Hub Location" to explore the competition among a large company and several medium-sized firms. They suggested that a leader company could lose a huge percentage of travelers regardless of competition strategies. Based on the Stackelberg hub location model. Sasaki [7] began to design a hub network, in which two companies compete over maximizing their profits. Sasaki et al. [8] proposed a model for the hub location in a competitive environment. Whereas Sasaki and Fukushima [6] and Sasaki [7] allowed only using one hub in the path of flow passing from origin to destination, they studied more than one hub in their model. Eiselt and Marianov [9] proposed a hub location problem in a competitive environment in which customers based on the parameters of the ticket price and flight time used the gravity-like function to select airline. They implemented their model for the follower player as a new entrant player to the Australian Postal network.

Code Sharing Agreement is a commercial agreement in air transportation, in which two or more airlines share a common flight. In this paper, we consider the alliance between Mahan Airline, Iran Air, and Aseman Airline using Code Sharing Agreement. Lin [10] assessed the economic effects of alliance and cooperation between an international airline and a local airline. He suggested that two Stackelberg equilibrium points were achievable when a set of allied airlines was identified as the leader (follower) player. Lin [10] also demonstrated that in addition to an increase in the social welfare of travelers, the alliance might reduce international traveler excess and local direct travelers. Zou and Chen [11] investigated the effects of code sharing and global alliance on the performance of airlines simultaneously. The results showed that the profit margin of an airline was positively associated with the number of partners in code sharing. Yimga [12] investigated the relationship between alliances and on-time performance. He found evidence that code-sharing alliances improve the on-time performance rate.

Nikoofal and Sadjadi [13] used Bertimas and Sim's approach [5] to propose the robust model of the median hub location problem with uncertain travel cost. Taking advantage of Bertsimas and Sim's approach [5], GhaffariNasab et al. [14] proposed a robust model for the capacitated hub location problem (single allocationmultiple allocation) in which the demand change was uncertain. They used uncertain demand only for capacity constraints and supposed that the objective function of demand is certain. Zetina et al. [15] proposed a robust counterpart for multiple hub location problem in which (like Bertsimas and Sim's approach [5]) the level of conservativeness was controlled by using uncertainty budget. The problem was modeled in three modes: uncertain transportation cost, uncertain demand, and both simultaneously. 
A variety of are reviewed in articles. Some of the most attractive solving methods are as follows:

Tavakkoli-Moghadam et al. [16] developed a multiobjective mathematical model for a capacitated single hub location problem to determine the location and the capacity of hub node. The model is solved by a multiobjective imperialist competitive algorithm. The authors minimized the total cost of the networks and minimize the maximum travel time between nodes.

Ghodratnama et al. [17] presented a new mathematical model for a p-hub location-allocation problem and compared three Meta-heuristics a namely genetic algorithm, particle swarm optimization, and simulated annealing to solve it. Bashiri and Rezanezhad [18] presented a multi-objective model for uncapacitated single allocation and used the $\varepsilon$-constraint algorithm for small size instances and NSGA-II to obtain Pareto solutions. The model aims to minimize the total investment and transportation costs, minimizes the maximum traveling time between the pair of nodes, maximizes the total reliability of available paths and forces allocating near nodes to more reliable hubs. Alizadeh et al. [19] presented a new multi-objective mathematical model for a capacitated hub maximal covering problem. They solved the model by nondominated sorting genetic algorithm (NSGA-II) and nondominated ranking genetic algorithm (NRGA). Karimi, et al. [20] formulated the multi-modal single allocation capacitated p-hub covering problem. They presented a heuristic based on the taboo search algorithm to solve the problem.

\section{PROBLEMS STATEMENT AND MATHEMATICAL MODEL}

In this section, we developed the model of Marianov et al. [1] for an alliance of airlines with a lower market share. The notations used in the proposed deterministic model are as follows:

Sets:

$$
\begin{aligned}
& N_{i j}^{1}=\left\{k \in K \mid c_{i k}+c_{k j}<C_{i j}, t_{i k}+t_{k}+t_{k j} \geq T_{i j}, V_{k}<V\right\} \\
& N_{i j}^{2}=\left\{k \in K \mid c_{i k}+c_{k j}<C_{i j}, t_{i k}+t_{k}+t_{k j} \geq T_{i j}, V_{k} \geq V\right\} \\
& M_{i j}^{1}=\left\{k \in K \mid c_{i k}+c_{k j} \geq C_{i j}, t_{i k}+t_{k}+t_{k j}<T_{i j}, V_{k}<V\right\} \\
& M_{i j}^{2}=\left\{k \in K \mid c_{i k}+c_{k j} \geq C_{i j}, t_{i k}+t_{k}+t_{k j}<T_{i j}, V_{k} \geq V\right\} \\
& P_{i j}^{1}=\left\{k \in K \mid c_{i k}+c_{k j}<C_{i j}, t_{i k}+t_{k}+t_{k j}<T_{i j}, V_{k}<V\right\} \\
& P_{i j}^{2}=\left\{k \in K \mid c_{i k}+c_{k j}<C_{i j}, t_{i k}+t_{k}+t_{k j}<T_{i j}, V_{k} \geq V\right\} \\
& K^{\prime}=N_{i j}^{1} \cup N_{i j}^{2} \quad K^{\prime \prime}=M_{i j}^{1} \cup M_{i j}^{2} \\
& K^{\prime \prime \prime}=P_{i j}^{1} \cup P_{i j}^{2} \quad K=K^{\prime} \cup K^{\prime \prime} \cup K^{\prime \prime \prime}
\end{aligned}
$$

\begin{tabular}{|c|c|}
\hline$c_{i k}$ & Ticket price for passing a traveller from $i$ to $k$ \\
\hline$c_{k j}$ & Ticket price for passing a traveller from $k$ to $j$ \\
\hline$C_{i j}$ & $\begin{array}{l}\text { Total airfare of passing a traveller from } \\
\text { itojthrough leader hub(s) }\end{array}$ \\
\hline$t_{k}$ & Average time delay for transferring a traveller in $k$ \\
\hline$t_{i k}$ & Time needed to pass travelers from $i$ to $k$ \\
\hline$t_{k j}$ & Time needed to pass travelers from $k$ to $j$ \\
\hline$T_{i j}$ & Total time needed to pass from $i$ to $j$ \\
\hline$W_{i j}$ & $\begin{array}{l}\text { Total flow passing from } i \text { to } j \text { which has already } \\
\text { moved by the leader }\end{array}$ \\
\hline$\Gamma_{k}$ & Capacity of $k$ \\
\hline$E_{k}$ & Minimum passing flow to select $k$ as the hub \\
\hline$V_{k}$ & Service quality of $k$ controlled by follower airline \\
\hline$V$ & $\begin{array}{l}\text { Average service quality of airports controlled by } \\
\text { leader airline }\end{array}$ \\
\hline$U_{f}$ & Quality of follower airline \\
\hline$U_{l}$ & Quality of leader airline \\
\hline$S_{f}$ & Safety of follower airline \\
\hline$S_{l}$ & Safety of leader airline \\
\hline$t_{f}$ & $\begin{array}{l}\text { Average travel time delay transferring a traveller } \\
\text { by follower airline }\end{array}$ \\
\hline$t_{l}$ & $\begin{array}{l}\text { Average travel time delay transferring a traveller } \\
\text { by leader airline }\end{array}$ \\
\hline$f_{c}$ & Importance of cost for a traveller \\
\hline$f_{t}$ & Importance of time for a traveller \\
\hline$f_{q}$ & $\begin{array}{l}\text { Importance of service quality of airport for a } \\
\text { traveller }\end{array}$ \\
\hline$M$ & Large positive number \\
\hline$p_{1}, p_{2}$ & Reduction factor of $W_{i j}$ for sets $N_{i j}^{1}, N_{i j}^{2}$ \\
\hline$q_{1}, q_{2}$ & Reduction factor of $W_{i j}$ for sets $M_{i j}^{1}, M_{i j}^{2}$ \\
\hline$r_{1}, r_{2}$ & Reduction factor of $W_{i j}$ for sets $P_{i j}^{1}, P_{i j}^{2}$ \\
\hline$\beta_{1}, \beta_{2}$ & Discount factor for ticket price \\
\hline$\gamma_{1}, \gamma_{2}$ & Discount factor for ticket price \\
\hline
\end{tabular}

\section{Indices:}

$$
\begin{array}{l|l}
I, i & \text { Set and counter of origins } \\
J, j & \text { Set and counter of destinations }
\end{array}
$$

$K, k \quad$ Set and counter of all candidate hubs

\section{Parameters:}

\section{Decision variables:}

\begin{tabular}{l|l}
$h_{k}$ & $\begin{array}{l}\text { Equals } 1 \text { if } k \text { is selected as a hub, otherwise, Equals } \\
x_{k j}^{i 1}\end{array}$ \\
$x_{k j}^{i 2}$ & Amount of flow passing from $i$ toj through $k \in N_{i j}^{1}$ \\
$y_{k j}^{i 1}$ & Amount of flow passing from $i$ to $j$ through $k \in N_{i j}^{2}$ \\
$y_{k j}^{i 2}$ & Amount of flow passing from $i$ toj through $k \in M_{i j}^{1}$ \\
$z_{k j}^{i 1}$ & Amount flow of passing from $i$ to $j$ through $k \in P_{i j}^{1}$ \\
$z_{k j}^{i 2}$ & Amount flow of passing from $i$ toj through $k \in P_{i j}^{2}$
\end{tabular}




\section{1. Objective Function}

$$
\begin{aligned}
& z=\max \sum_{k \in K} \sum_{i \in I-K} \sum_{j \neq i \in J-K} \sum_{s=1}^{2}\left(x_{k j}^{i s}+y_{k j}^{i s}+\right. \\
& \left.z_{k j}^{i s}\right)\left(\gamma_{1} c_{i k}+\beta_{1} c_{k j}\right)+\sum_{i \in I-K} \sum_{j \in K} \sum_{s=1}^{2}\left(x_{j j}^{i s}+y_{j j}^{i s}+\right. \\
& \left.z_{j j}^{i s}\right)\left(\gamma_{2} c_{i j}\right)+\sum_{k \in K} \sum_{j \in J-K} \sum_{s=1}^{2}\left(x_{k j}^{k S}+y_{k j}^{k S}+\right. \\
& \left.z_{k j}^{k S}\right)\left(\beta_{2} c_{k j}\right)\left(\beta_{1} \leq \gamma_{1} \leq \beta_{2} \leq \gamma_{2}\right)
\end{aligned}
$$

The first term of the objective Function (1) represents the total income from selling tickets to travelers passing from origin $i$ (per each origin) to destination $j$ (to all destinations) through hubs $k$ of the follower airline. Accordingly, none of the follower airline's hubs can be the origin or destination of travel. The second term of the objective Function (1) represents the total income from selling tickets to travelers who their destinations are one of the hubs of follower airline. The third term of the objective Function (1) represents the total income from selling tickets to travelers who their origins are one of the hubs of follower airline. Here is the reason why there is a relationship between ticket prices in the objective Function (1) such that $\beta_{1} \leq \gamma_{1} \leq \beta_{2} \leq \gamma_{2}$. When travelers use any other origin of follower airline's hubs to reach any other destination of follower airline's hubs, their profitability for the airline will be higher, and they deserve more discounts. In other words, $\beta_{1}, \gamma_{1} \leq \gamma_{2}$ and $\beta_{1}, \gamma_{1} \leq \gamma_{2}$. In this way, because travelers use follower airline's hub in the second part of their travel, airport costs are decreased. Also, because travelers from different origins aim to reach the same destination, taking advantage of a bigger airplane with a lower ticket price seems more logical; therefore $\beta_{1} \leq \gamma_{1}$. When the origin or destination is one of the follower airline's hubs, the amount of discount is lower due to shorter travel. So, when the origin is follower airline's hub, we have $\beta_{2} \leq$ $\gamma_{2}$. Because airport costs are lower, travelers from other origins are going to reach the aforementioned destination.

\section{2. Constraints Constraints (2) to (7) show} that the flow is established when a hub exists

$$
\begin{aligned}
& \sum_{k \in N_{i j}^{1}} x_{k j}^{i 1} \leq W_{i j} * p_{1} \forall i \in I, j \in J \\
& \sum_{k \in N_{i j}^{2}} x_{k j}^{i 2} \leq W_{i j} * p_{2} \forall i \in I, j \in J \\
& \sum_{k \in M_{i j}^{1}} y_{k j}^{i 1} \leq W_{i j} * q_{1} \forall i \in I, j \in J \\
& \sum_{k \in M_{i j}^{2}} y_{k j}^{i 2} \leq W_{i j} * q_{2} \forall i \in I, j \in J \\
& \sum_{k \in P_{i j}^{1}} z_{k j}^{i 1} \leq W_{i j} * r_{1} \forall i \in I, j \in J \\
& \sum_{k \in P_{i j}^{2}} z_{k j}^{i 2} \leq W_{i j} * r_{2} \forall i \in I, j \in J
\end{aligned}
$$

According to Constraint (8), the maximum flow passing from all origins to all destinations through follower airline hubs is equal to $\left[W_{i j}\left(\frac{U_{f}}{U_{l}}\right)\left(\frac{s_{f}}{s_{l}}\right)\left(\frac{t_{l}}{t_{f}}\right)\right]$. Also, this constraint shows multiple allocations.

$$
\begin{aligned}
& \sum_{s=1}^{2}\left(\sum_{k \in K} x_{k j}^{i s}+\sum_{k \in K} y_{k j}^{i s}+\sum_{k \in K} z_{k j}^{i s}\right) \leq \\
& W_{i j}\left(\frac{U_{f}}{U_{l}}\right)\left(\frac{S_{f}}{s_{l}}\right)\left(\frac{t_{l}}{t_{f}}\right) \forall i, j
\end{aligned}
$$

Constraint (9) shows that the maximum incoming flow to hub $k$ equals $\Gamma_{k}$.

$$
\sum_{i \in I} \sum_{j \neq i \in J} \sum_{s=1}^{2} x_{k j}^{i s}+y_{k j}^{i s}+z_{k j}^{i s} \leq \Gamma_{k} h_{k} \forall k \in K
$$

Constraint (10) shows that the minimum flow passing for using spoke $k$ as a hub is equal to $E_{k}$.

$$
\begin{aligned}
& E_{k}-\sum_{i \in I} \sum_{j \in J} \sum_{s=1}^{2}\left(x_{k j}^{i s}+y_{k j}^{i s}+z_{k j}^{i s}\right) \leq \\
& M\left(1-h_{k}\right) \forall k \in K
\end{aligned}
$$

Constraint (11) guarantees that for each origin $i$ and destination $j$, only variables $x_{k j}^{i s}$ related to airports $k$ in set $K$ 'can be non-zero, and the remaining variables are equal to zero.

$$
x_{k j}^{i S}=0 \forall i \in I, j \in J ; \forall s=1,2 ; k \in K-K^{\prime}
$$

Constraint (12) guarantees that for each origin $i$ and destination $j$, only variables $y_{k j}^{i s}$ related to airports $k$ in set $K "$ can be non-zero, and the remaining variables are equal to zero.

$$
y_{k j}^{i s}=0 \forall i \in I, j \in J ; \forall s=1,2 ; k \in K-K^{\prime \prime}
$$

Constraint (13) guarantees that for each origin $i$ and destinationj, only variables $z_{k j}^{i s}$ related to airports $k$ in set $K^{\prime \prime}$ can be non-zero, and the remaining variables are equal to zero.

$$
z_{k j}^{i s}=0 \forall i \in I, j \in J ; \forall s=1,2 ; k \in K-K^{\prime \prime}
$$

Constraints (14) and (15) show the domain of variables.

$$
\begin{aligned}
& h_{k} \in\{0,1\} \forall k \in K \\
& x_{k j}^{i 1}, x_{k j}^{i 2}, y_{k j}^{i 1}, y_{k j}^{i 2}, z_{k j}^{i 1}, z_{k j}^{i 2} \in I^{+} \cup\{0\} \forall i \in I, j \in \\
& J, k \in K
\end{aligned}
$$

\section{3. Determining the Importance of Travel Time, Travel Cost and Service Quality $f_{c}, f_{t}$ and $f_{q}$} represent the degree of importance of the parameters of travel time, travel cost and service quality of hubs for travelers. To determine them, we used data gathered by questionnaires from air travelers with different income levels, ages, and travel purposes. Also, we introduced the interpolation function of importance for time and cost in air travels by using a multivariate Lagrange interpolation method. Lagrange interpolation function with $m$ variables and degree $n$ is defined as Equation (16) ([21]):

$$
f\left(X_{1}, X_{1}, \cdots, X_{m}\right)=\sum_{e_{i} .1 \leq n} \alpha_{e_{i}} X^{e_{i}}
$$

where $e_{i}=\left(e_{1 i}, \cdots, e_{m i}\right)$ and $\sum_{j=1}^{m} e_{j i}=1$.

In order to uniquely determine $f$, we require $\rho=$ $\left(\begin{array}{c}n+m \\ n\end{array}\right)$ independent points in Equation (17). 


$$
\begin{aligned}
& \left(x_{1, i}, \cdots, x_{m, i}, f_{i}\right) \in R^{m+1} \quad, 1 \leq i \leq \rho, f_{i}= \\
& f\left(x_{1, i}, \cdots, x_{m, i}\right)
\end{aligned}
$$

By calculating the degree of importance of travel time, travel cost and service quality of airport and by taking quality, safety and travel time delay of the follower and leader airline into account, parameters $p_{1}, p_{2}, q_{1}, q_{2}$ and $r_{1}, r_{2}$ are determined as Equation (18).

$$
\begin{aligned}
& p_{1}=f_{c} *\left(\frac{U_{f}}{U_{l}}\right) *\left(\frac{S_{f}}{S_{l}}\right) *\left(\frac{t_{l}}{t_{f}}\right) \\
& p_{2}=\left(f_{c}+f_{q}\right) *\left(\frac{U_{f}}{U_{l}}\right) *\left(\frac{S_{f}}{S_{l}}\right) *\left(\frac{t_{l}}{t_{f}}\right) \\
& q_{1}=f_{t} *\left(\frac{U_{f}}{U_{l}}\right) *\left(\frac{S_{f}}{S_{l}}\right) *\left(\frac{t_{l}}{t_{f}}\right) \\
& q_{2}=\left(f_{t}+f_{q}\right) *\left(\frac{U_{f}}{U_{l}}\right) *\left(\frac{S_{f}}{S_{l}}\right) *\left(\frac{t_{l}}{t_{f}}\right) \\
& r_{1}=\left(f_{c}+f_{t}\right) *\left(\frac{U_{f}}{U_{l}}\right) *\left(\frac{S_{f}}{S_{l}}\right) *\left(\frac{t_{l}}{t_{f}}\right) \\
& r_{2}=\left(f_{c}+f_{t}+f_{q}\right) *\left(\frac{U_{f}}{U_{l}}\right) *\left(\frac{S_{f}}{S_{l}}\right) *\left(\frac{t_{l}}{t_{f}}\right)
\end{aligned}
$$

\section{ROBUST OPTIMIZATION}

In this section, we presented a robust counterpart of the model proposed in section 3 by employing Bertsimas and Sim's approach [5]. Given the impact of seasonal changes (such as travel in the high season) and weather conditions on the number of travelers, we consider $w_{i j}$ as uncertain. The values of the uncertain parameters $\widetilde{w}_{i j}$ are selected based on a symmetric distribution in the interval $\left[w_{i j}-\hat{w}_{i j}, w_{i j}+\hat{w}_{i j}\right]$ with an average of $w_{i j}$.

The notations used in the proposed robust model are as follows:

\section{Sets:}

$$
\begin{aligned}
& F^{\text {const }}=\left\{(i, j) \mid W_{i j} \text { hasnoise }\left(\hat{W}_{i j}>0\right)\right\}, \text { const }=1, \ldots, 7 \\
& \cup_{\text {onst }=1}^{7}\left|F^{\text {const }}\right| \leq|I| \times|J|
\end{aligned}
$$

\section{Parameters:}

$$
\begin{array}{l|l}
\hat{W}_{i j} & \text { Deviation from } w_{i j} \\
\Gamma_{i j}^{c o n s t} & \text { Number of uncertain parameters } w_{i j}
\end{array}
$$

\section{Decision variables:}

$$
\begin{array}{ll}
P_{i j f^{\text {const }}, Z_{i j}^{\text {const }}}^{\text {cons }} & \text { Dual auxiliary variables } \\
& \text { The variable defined to include right-hand } \\
H & \text { side vector } b \text { as a column of technical } \\
& \text { matrix } A
\end{array}
$$

4. 1. Robust Model Formulation

Given the fact that uncertain parameters $w_{i j}$ are included in
Constraints (2) to (8), we employed Bertsimas and Sim's approach [5] and by defining variable $\mathrm{H}$, we showed the above mentioned constraints in the form of Constraints (19) to (26):

$$
\begin{aligned}
& \sum_{k \in N_{i j}^{1}} x_{k j}^{i 1}-W_{i j}\left(f_{c}\right)\left(\frac{U_{f}}{U_{l}}\right)\left(\frac{S_{f}}{S_{l}}\right)\left(\frac{t_{l}}{t_{f}}\right) H \leq 0 \forall i \in I, j \in J \\
& \sum_{k \in N_{i j}^{2}} x_{k j}^{i 2}-W_{i j}\left(f_{c}+f_{q}\right)\left(\frac{U_{f}}{U_{l}}\right)\left(\frac{S_{f}}{S_{l}}\right)\left(\frac{t_{l}}{t_{f}}\right) H \leq 0 \\
& \forall i \in I, j \in J \\
& \sum_{k \in M_{i j}^{1}} y_{k j}^{i 1}-W_{i j}\left(f_{t}\right)\left(\frac{U_{f}}{U_{l}}\right)\left(\frac{S_{f}}{S_{l}}\right)\left(\frac{t_{l}}{t_{f}}\right) H \leq 0 \forall i \in I, j \in J \\
& \sum_{k \in M_{i j}^{2}} y_{k j}^{i 2}-W_{i j}\left(f_{t}+f_{q}\right)\left(\frac{U_{f}}{U_{l}}\right)\left(\frac{s_{f}}{s_{l}}\right)\left(\frac{t_{l}}{t_{f}}\right) H \leq 0 \\
& \forall i \in I, j \in J \\
& \sum_{k \in P_{i j}^{1}} z_{k j}^{i 1}-W_{i j}\left(f_{c}+f_{t}\right)\left(\frac{U_{f}}{U_{l}}\right)\left(\frac{s_{f}}{s_{l}}\right)\left(\frac{t_{l}}{t_{f}}\right) H \leq 0 \\
& \forall i \in I, j \in J \\
& \sum_{k \in P_{i j}^{2}} z_{k j}^{i 2} \leq W_{i j}\left(f_{c}+f_{t}+f_{q}\right)\left(\frac{U_{f}}{U_{l}}\right)\left(\frac{S_{f}}{s_{l}}\right)\left(\frac{t_{l}}{t_{f}}\right) H \leq 0 \\
& \forall i \in I, j \in J
\end{aligned}
$$

$0 \leq H \leq 1$

Given the fact that Constraints (19) to (26) are defined for each $i$ and each $j$, constraints itself subjects to maximum $i \times j$ constraints. Since each of those constraints had only ones $w_{i j}$, we got $\Gamma_{i j}^{\text {const }} \in$ $\left[0,\left|F^{\text {const }}\right|\right]$, where, $F^{\text {const }} \subseteq\{0,1\}$. Based on research by Bertsimas and Sim [5], Constraint (19) is turned into Constraint (27).

$$
\begin{aligned}
& \sum_{k \in N_{i j}^{1}} x_{k j}^{i 1}-W_{i j}\left(f_{c}\right)\left(\frac{U_{f}}{U_{l}}\right)\left(\frac{S_{f}}{S_{l}}\right)\left(\frac{t_{l}}{t_{f}}\right) H+ \\
& \max _{\forall(i, j) \in F^{1}}\left\{\begin{array}{l}
\left\lfloor\Gamma_{i j}^{1}\right\rfloor \hat{w}_{i j}\left(f_{c}\right)\left(\frac{U_{f}}{U_{l}}\right)\left(\frac{S_{f}}{s_{l}}\right)\left(\frac{t_{l}}{t_{f}}\right) H \\
+\left(\Gamma_{i j}^{1}-\left\lfloor\Gamma_{i j}^{1}\right\rfloor\right) \hat{w}_{i j}\left(f_{c}\right)\left(\frac{U_{f}}{U_{l}}\right)\left(\frac{S_{f}}{S_{l}}\right)\left(\frac{t_{l}}{t_{f}}\right) H
\end{array}\right\} \leq 0 \\
& \forall i \in I, j \in J
\end{aligned}
$$

Similarly, Constraints (20) to (26) are modified.

Then, by proving the theorem 1 , we showed that Constraint (27) is equal to a linear optimization problem. The way of proving the theorem proving for other similar constraints was the same.

Theorem 1. For providing $H^{*}$, Function (28):

$$
\begin{aligned}
& \beta_{1}\left(H^{*}, \Gamma_{i j}^{1}\right)= \\
& \max _{\forall(i, j) \in F^{1}}\left\{\begin{array}{l}
\left\lfloor\Gamma_{i j}^{1}\right\rfloor \hat{w}_{i j}\left(f_{c}\right)\left(\frac{U_{f}}{U_{l}}\right)\left(\frac{s_{f}}{s_{l}}\right)\left(\frac{t_{l}}{t_{f}}\right) H^{*} \\
+\left(\Gamma_{i j}^{1}-\left\lfloor\Gamma_{i j}^{1}\right\rfloor\right) \hat{w}_{i j}\left(f_{c}\right)\left(\frac{U_{f}}{U_{l}}\right)\left(\frac{s_{f}}{s_{l}}\right)\left(\frac{t_{l}}{t_{f}}\right) H^{*}
\end{array}\right\}
\end{aligned}
$$


is equal to the linear programming problem below.

$$
\begin{gathered}
\beta_{1}\left(H^{*}, \Gamma_{i j}^{1}\right)=\max \quad \hat{w}_{i j}\left(f_{c}\right)\left(\frac{U_{f}}{U_{l}}\right)\left(\frac{S_{f}}{S_{l}}\right)\left(\frac{t_{l}}{t_{f}}\right) H^{*} z_{i j} \\
\text { s.t. } \quad z_{i j} \leq \Gamma_{i j}^{1} \\
0 \leq z_{i j} \leq 1 \forall(i, j) \in F^{1}
\end{gathered}
$$

Proof. We proved the theorem in two cases:

Case 1: $\Gamma_{i j}^{1}$ is an integer.

So $\Gamma_{i j}^{1} \in[0,1]$, then $\Gamma_{i j}^{1}=1$. In this case, $\beta_{1}\left(H^{*}, \Gamma_{i j}^{1}\right)$ is equal to:

$$
\begin{aligned}
& \beta_{1}\left(H^{*}, \Gamma_{i j}^{1}\right)= \\
& \max _{\forall(i, j) \in F^{1}}\left\{\hat{w}_{i j}\left(f_{c}\right)\left(\frac{U_{f}}{U_{l}}\right)\left(\frac{S_{f}}{S_{l}}\right)\left(\frac{t_{l}}{t_{f}}\right) H^{*}\right\}= \\
& \hat{w}_{i j}\left(f_{c}\right)\left(\frac{U_{f}}{U_{l}}\right)\left(\frac{S_{f}}{S_{l}}\right)\left(\frac{t_{l}}{t_{f}}\right) H^{*}
\end{aligned}
$$

Because the optimization problem was a maximization one, then, we considered $z_{i j}=\Gamma_{i j}^{1}=1$. Therefore, the problem can become feasible and the optimal value of objective function is equal to:

$$
\hat{w}_{i j}\left(f_{c}\right)\left(\frac{U_{f}}{U_{l}}\right)\left(\frac{s_{f}}{s_{l}}\right)\left(\frac{t_{l}}{t_{f}}\right) H^{*}
$$

Hence, in this case, Equation (29) is correct.

Case 2: $\Gamma_{i j}^{1}$ is non-integer.

So $\Gamma_{i j}^{1} \in[0,1]$, then $\left\lfloor\Gamma_{i j}^{1}\right\rfloor=0$. In this case, $\beta_{1}\left(H^{*}, \Gamma_{i j}^{1}\right)$ is equal to:

$$
\begin{aligned}
& \beta_{1}\left(H^{*}, \Gamma_{i j}^{1}\right)=\max _{\forall(i, j) \in F^{1}}\left\{\left(\Gamma_{i j}^{1}-\right.\right. \\
& \left.0) \hat{w}_{i j}\left(f_{c}\right)\left(\frac{U_{f}}{U_{l}}\right)\left(\frac{S_{f}}{s_{l}}\right)\left(\frac{t_{l}}{t_{f}}\right) H^{*}\right\}= \\
& \Gamma_{i j}^{1} \hat{w}_{i j}\left(f_{c}\right)\left(\frac{U_{f}}{U_{l}}\right)\left(\frac{s_{f}}{s_{l}}\right)\left(\frac{t_{l}}{t_{f}}\right) H^{*}
\end{aligned}
$$

Because the optimization problem was a maximization one, then we considered $z_{i j}=\Gamma_{i j}^{1}$. Therefore, the problem can become feasible and the optimal value of objective function is equal to:

$$
\Gamma_{i j}^{1} \hat{w}_{i j}\left(f_{c}\right)\left(\frac{U_{f}}{U_{l}}\right)\left(\frac{s_{f}}{s_{l}}\right)\left(\frac{t_{l}}{t_{f}}\right) H^{*}
$$

Hence, in this case, Equation (29) is correct.

By using theorem 1 and taking advantage of Strong duality theorem, it can be shown that the robust counterpart of robust model presented in section 3 is as following:

$$
(1,10-15)
$$

$$
\begin{aligned}
& \sum_{k \in N_{i j}^{1}} x_{k j}^{i 1}-W_{i j}\left(f_{c}\right)\left(\frac{U_{f}}{U_{l}}\right)\left(\frac{S_{f}}{S_{l}}\right)\left(\frac{t_{l}}{t_{f}}\right) H+z_{i j}^{1} \Gamma_{i j}^{1}+ \\
& \sum_{f^{1} \in F^{1}} P_{i j f^{1}}^{1} \leq 0 \forall i \in I, j \in J \\
& \sum_{k \in N_{i j}^{2}} x_{k j}^{i 2}-W_{i j}\left(f_{c}+f_{q}\right)\left(\frac{U_{f}}{U_{l}}\right)\left(\frac{S_{f}}{S_{l}}\right)\left(\frac{t_{l}}{t_{f}}\right) H+z_{i j}^{2} \Gamma_{i j}^{2}+ \\
& \sum_{f^{2} \in F^{2}} P_{i j f^{2}}^{2} \leq 0 \quad \forall i \in I, j \in J \\
& \sum_{k \in M_{i j}^{1}} y_{k j}^{i 1}-W_{i j}\left(f_{t}\right)\left(\frac{U_{f}}{U_{l}}\right)\left(\frac{S_{f}}{S_{l}}\right)\left(\frac{t_{l}}{t_{f}}\right) H+z_{i j}^{3} \Gamma_{i j}^{3}+
\end{aligned}
$$

$$
\begin{aligned}
& \sum_{f^{3} \in F^{3}} P_{i j f^{3}}^{3} \leq 0 \forall i \in I, j \in J \\
& \sum_{k \in M_{i j}^{2}} y_{k j}^{i 2}-W_{i j}\left(f_{t}+f_{q}\right)\left(\frac{U_{f}}{U_{l}}\right)\left(\frac{S_{f}}{S_{l}}\right)\left(\frac{t_{l}}{t_{f}}\right) H+z_{i j}^{4} \Gamma_{i j}^{4}+ \\
& \sum_{f^{4} \in F^{4}} P_{i j f^{4}}^{4} \leq 0 \quad \forall i \in I, j \in J \\
& \sum_{k \in P_{i j}^{1}} z_{k j}^{i 1}-W_{i j}\left(f_{c}+f_{t}\right)\left(\frac{U_{f}}{U_{l}}\right)\left(\frac{S_{f}}{S_{l}}\right)\left(\frac{t_{l}}{t_{f}}\right) H+z_{i j}^{5} \Gamma_{i j}^{5}+ \\
& \sum_{f^{5} \in F^{5}} P_{i j f^{5}}^{5} \leq 0 \quad \forall i \in I, j \in J \\
& \sum_{k \in P_{i j}^{2}} z_{k j}^{i 2}-W_{i j}\left(f_{c}+f_{t}+f_{q}\right)\left(\frac{U_{f}}{U_{l}}\right)\left(\frac{s_{f}}{s_{l}}\right)\left(\frac{t_{l}}{t_{f}}\right) H \\
& +z_{i j}^{6} \Gamma_{i j}^{6}+\sum_{f^{6} \in F^{6}} P_{i j f^{6}}^{6} \leq 0 \quad \forall i \in I, j \in J \\
& \sum_{s=1}^{2}\left(\sum_{k \in K} x_{k j}^{i s}+\sum_{k \in K} y_{k j}^{i s}+\right. \\
& \left.\sum_{k \in K} z_{k j}^{i s}\right)-W_{i j}\left(\frac{U_{f}}{U_{l}}\right)\left(\frac{S_{f}}{S_{l}}\right)\left(\frac{t_{l}}{t_{f}}\right) H+z_{i j}^{7} \Gamma_{i j}^{7}+ \\
& \sum_{f^{7} \in F^{7}} P_{i j f^{7}}^{7} \leq 0 \quad \forall i, j \\
& \hat{W}_{i j}\left(\left(f_{c}\right)\left(\frac{U_{f}}{U_{l}}\right)\left(\frac{S_{f}}{S_{l}}\right)\left(\frac{t_{l}}{t_{f}}\right)\right) H \leq z_{i j}^{1}+P_{i j f^{1}}^{1} \\
& \forall i \in I, j \in J, f^{1} \in F^{1} \\
& \hat{W}_{i j}\left(\left(f_{c}+f_{q}\right)\left(\frac{U_{f}}{U_{l}}\right)\left(\frac{S_{f}}{S_{l}}\right)\left(\frac{t_{l}}{t_{f}}\right)\right) H \leq z_{i j}^{2}+P_{i j f^{2}}^{2} \\
& \forall i \in I, j \in J, f^{2} \in F^{2} \\
& \hat{W}_{i j}\left(\left(f_{t}\right)\left(\frac{U_{f}}{U_{l}}\right)\left(\frac{S_{f}}{S_{l}}\right)\left(\frac{t_{l}}{t_{f}}\right)\right) H \leq z_{i j}^{3}+P_{i j f^{3}}^{3} \\
& \forall i \in I, j \in J, f^{3} \in F^{3} \\
& \hat{W}_{i j}\left(\left(f_{t}+f_{q}\right)\left(\frac{U_{f}}{U_{l}}\right)\left(\frac{S_{f}}{S_{l}}\right)\left(\frac{t_{l}}{t_{f}}\right)\right) H \leq z_{i j}^{4}+P_{i j f^{4}}^{4} \\
& \forall i \in I, j \in J, f^{4} \in F^{4} \\
& \hat{W}_{i j}\left(\left(f_{c}+f_{t}\right)\left(\frac{U_{f}}{U_{l}}\right)\left(\frac{S_{f}}{S_{l}}\right)\left(\frac{t_{l}}{t_{f}}\right)\right) H \leq z_{i j}^{5}+P_{i j f^{5}}^{5} \\
& \forall i \in I, j \in J, f^{5} \in F^{5} \\
& \hat{W}_{i j}\left(\left(f_{c}+f_{t}+f_{q}\right)\left(\frac{U_{f}}{U_{l}}\right)\left(\frac{s_{f}}{S_{l}}\right)\left(\frac{t_{l}}{t_{f}}\right)\right) H \leq z_{i j}^{6}+ \\
& P_{i j f^{6}}^{6} \quad \forall i \in I, j \in J, f^{6} \in F^{6} \\
& \hat{W}_{i j}\left(\left(\frac{U_{f}}{U_{l}}\right)\left(\frac{S_{f}}{S_{l}}\right)\left(\frac{t_{l}}{t_{f}}\right)\right) H \leq z_{i j}^{7}+P_{i j f^{7}}^{7} \\
& \forall i \in I, j \in J, f^{7} \in F^{7} \\
& z_{i j}^{\text {const }}, P_{i j f^{c o n s t}}^{\text {const }} \geq 0 \\
& \forall i \in I, j \in J, f^{\text {const }} \in F^{\text {const }}, \text { const } \in\{1,2, \cdots, 7\}
\end{aligned}
$$

\section{COMPUTATIONAL RESULTS}

In this section, we studied a case to examine the proposed model in designing optimal locations of hubs for a follower airline, in which Emirate Airline was the leader, and an alliance consisted of Mahan Airline, Iran Air, and 
Aseman Airline could play the role of follower airlines. Also, the results are compared with the results of the Marianov et al. [1] model implementation.

By using the results of 400 distributed questionnaires, can be shown that:

$$
f_{t}=47 \%, f_{c}=30 \%, f_{q}=23 \%
$$

According to Equation (35) and assuming that $\frac{U_{f}}{U_{l}}=0.4$, $\frac{s_{f}}{s_{l}}=0.2$ and $\frac{t_{l}}{t_{f}}=0.25$, values of parameters $p_{1}, p_{2}, q_{1}, q_{2}, r_{1}$ and $r_{2}$ are presented as Equation (36) by substituting the above-mentioned values into Equation (18):

$$
\begin{aligned}
& p_{1}=0.006, p_{2}=0.011 \\
& q_{1}=0.009, q_{2}=0.014 \\
& r_{1}=0.015, r_{2}=0.02
\end{aligned}
$$

The data needed for the implementation of the model can be found in Appendix A.

\section{1. Implementation of Deterministic Model} Based on the above-mentioned information, we implemented the model with values $\beta_{1}=1$ and $\gamma_{1}=1$. The optimal value of the objective function was $Z^{*}=$ $889,333 \$$. Whereas the optimal value of Marianov et al. [1] model is $Z^{*}=848,566 \$$. The optimal solution is achieved by using the GAMS software and the Baron solving method. Therefore, it can be concluded that the location of hubs in this paper leads to a significant increase in the incomes of the follower airline. Table 1 shows the share of follower airline's hubs from leader airline's travelers.

According to Table 1, in our mode Imam Khomeini and Mashhad airports with 627 and 328 travelers had the largest international traffics among Iranian airports, respectively and in Marianov et al. [1] model Imam Khomeini and Isfahan airports with 485 and 316 travelers had the largest international traffics among Iranian airports, respectively.

Considering the $\$ 859$ as the average travel cost per passenger, it can be concluded that the transportation cost of 138 passengers (the difference between 8,460 and 8,322 ) is $\$ 118,542$. Thus, transporting 8,322 passengers via the Mariano et al. [1] network would result in $\$ 5,084,192$ in revenue for the follower airline. As a result, it can be concluded that by exploiting the model presented in this paper as well as using the follower airline for passenger transportation, an extra $\$ 44$ per passenger would be generated in revenue for the follower airline.

As shown in Table 1, in our model, nearly $2 \%$ of 75,710 travelers of leader airline (1,467 travelers) were attracted by hubs of follower airline. Based on information about Emirate Airline, it could be said that the cost of passing existing travelers was $\$ 1,252,088$. Therefore, we concluded that the alliance of Iranian airlines and using their competitive edge not only lead to an increase in their market share by $\$ 889,333$ but also decreased the ticket costs for travelers by 362,775 dollars. In fact, if $\beta_{1}=1$ and $\gamma_{1}=1$, on average, there would be about $\$ 247$ reduction in ticket price per traveler. Furthermore, it could be said that the time needed to move all existing travelers (in Table 1) by Emirate Airline was about 21,000 hours, while this time for an alliance of Iranian airlines was about 18,607 hours. In fact, when we use the alliance of Iranian airlines and

\begin{tabular}{|c|c|c|c|c|c|c|c|c|c|c|}
\hline \multirow{2}{*}{$\begin{array}{l}\text { Number of } \\
\text { travelers }\end{array}$} & \multicolumn{2}{|r|}{$j_{1}$} & \multicolumn{2}{|r|}{$\boldsymbol{j}_{2}$} & \multicolumn{2}{|r|}{$j_{3}$} & \multicolumn{2}{|r|}{$j_{4}$} & \multicolumn{2}{|r|}{$j_{5}$} \\
\hline & Our paper & Marianov & Our paper & Marianov & Our paper & Marianov & Our paper & Marianov & Our paper & Marianov \\
\hline \multirow[t]{2}{*}{$i_{1}$} & $38\left(k_{1}\right)$ & $43\left(k_{4}\right)$ & $32\left(k_{1}\right)$ & $47\left(k_{3}\right)$ & \multirow[t]{2}{*}{$14\left(k_{8}\right)$} & $14\left(k_{4}\right)$ & $\begin{array}{l}38\left(k_{1}\right) \\
26\left(k_{5}\right)\end{array}$ & $57\left(k_{4}\right)$ & \multirow[t]{2}{*}{$81\left(k_{1}\right)$} & $24\left(k_{5}\right)$ \\
\hline & $43\left(k_{6}\right)$ & & $36\left(k_{6}\right)$ & $20\left(k_{4}\right)$ & & $13\left(k_{6}\right)$ & $17\left(k_{8}\right)$ & $24\left(k_{5}\right)$ & & $57\left(k_{6}\right)$ \\
\hline \multirow{2}{*}{$i_{2}$} & $24\left(k_{2}\right)$ & $55\left(k_{1}\right)$ & $25\left(k_{1}\right)$ & $25\left(k_{1}\right)$ & \multirow{2}{*}{$14\left(k_{2}\right)$} & \multirow{2}{*}{$14\left(k_{1}\right)$} & $38\left(k_{2}\right)$ & $38\left(k_{1}\right)$ & $36\left(k_{1}\right)$ & $20\left(k_{5}\right)$ \\
\hline & $57\left(k_{5}\right)$ & $8\left(k_{3}\right)$ & $29\left(k_{2}\right)$ & $29\left(k_{3}\right)$ & & & $43\left(k_{8}\right)$ & $43\left(k_{4}\right)$ & $32\left(k_{2}\right)$ & $47\left(k_{6}\right)$ \\
\hline \multirow{2}{*}{$i_{3}$} & $43\left(k_{1}\right)$ & \multirow{2}{*}{$81\left(k_{3}\right)$} & $38\left(k_{2}\right)$ & $38\left(k_{1}\right)$ & $19\left(k_{2}\right)$ & $28\left(k_{1}\right)$ & \multirow{2}{*}{$81\left(k_{1}\right)$} & $38\left(k_{1}\right)$ & \multirow{2}{*}{$68\left(k_{1}\right)$} & $20\left(k_{4}\right)$ \\
\hline & $38\left(k_{5}\right)$ & & $43\left(k_{6}\right)$ & $43\left(k_{3}\right)$ & $21\left(k_{6}\right)$ & $30\left(k_{4}\right)$ & & $43\left(k_{4}\right)$ & & $47\left(k_{5}\right)$ \\
\hline \multirow{2}{*}{$i_{4}$} & \multirow{2}{*}{$28\left(k_{5}\right)$} & $19\left(k_{1}\right)$ & $19\left(k_{1}\right)$ & $12\left(k_{3}\right)$ & \multirow{2}{*}{$7\left(k_{6}\right)$} & \multirow{2}{*}{$10\left(k_{6}\right)$} & $21\left(k_{1}\right)$ & \multirow{2}{*}{$21\left(k_{1}\right)$} & $21\left(k_{1}\right)$ & $28\left(k_{5}\right)$ \\
\hline & & $22\left(k_{6}\right)$ & $21\left(k_{6}\right)$ & $28\left(k_{6}\right)$ & & & $19\left(k_{2}\right)$ & & $19\left(k_{2}\right)$ & $12\left(k_{5}\right)$ \\
\hline \multirow{3}{*}{$i_{5}$} & \multirow{2}{*}{$38\left(k_{2}\right)$} & \multirow{2}{*}{$57\left(k_{1}\right)$} & \multirow{2}{*}{$38\left(k_{1}\right)$} & $19\left(k_{1}\right)$ & & \multirow{2}{*}{$19\left(k_{1}\right)$} & $43\left(k_{1}\right)$ & & \multirow{2}{*}{$43\left(k_{1}\right)$} & \multirow{3}{*}{$57\left(k_{1}\right)$} \\
\hline & & & & $38\left(k_{4}\right)$ & $14\left(k_{6}\right)$ & & $20\left(k_{2}\right)$ & $57\left(k_{1}\right)$ & & \\
\hline & $43\left(k_{8}\right)$ & $24\left(k_{5}\right)$ & $43\left(k_{6}\right)$ & $24\left(k_{5}\right)$ & & $8\left(k_{4}\right)$ & $18\left(k_{8}\right)$ & & $38\left(k_{2}\right)$ & \\
\hline
\end{tabular}
their under-controlled hubs, travel time per traveler was decreased about 40 minutes on average.

TABLE 1. Number of travelers passing from the follower's airports 
Different modes are presented in Table 2 for discounting the ticket price via our model. These modes are provided for situations in which the airline's revenue is significantly higher than the revenue obtained via the Mariano et al. [1] model.

\section{2. Implementation of Robust Model}

Based on the above-mentioned information, we implemented the robust model in a situation where all parameters $w_{i j}$ were uncertain. By uncertainty of all parameters $w_{i j}$, we mean that:

$$
\Gamma_{i j}^{\text {const }}=1, \forall i \in I, j \in J, \text { const } \in\{1,2, \ldots, 7\}
$$

Also, we supposed that:

$$
\hat{w}_{i j}=0.1 * w_{i j} \forall i \in I, j \in J
$$

The optimal value of the objective function for $\beta_{1}=1$ and $\gamma_{1}=1$ was $Z^{*}=822,896 \$$.

Given the results of implementing the model in the mode of uncertainty, it was clear that Iranian airlines were accounted for $1.8 \%$ of travelers of Emirate Airline (i.e. 1,345 travelers). In fact, the number of travelers absorbed by Iranian airlines was decreased by 122 people in uncertainty mode, which in turn led to a decrease in revenue of Iranian airlines by $\$ 66,437$.

Based on information about Emirate Airline, it could be said that the cost of passing existing travelers was $\$ 1,252,088$. Therefore, we concluded that an alliance of Iranian airlines and using their competitive edge not only lead to an increase in their market share by $\$ 822,896$ but also decreased the ticket costs for travelers by $\$ 429,192$. In fact, if $\beta_{1}=1$ and $\gamma_{1}=1$, on average, there would be about $\$ 364$ reduction in ticket price per traveler. Furthermore, it could be said that the time needed to move all existing travelers (in Table 1) by Emirate Airline was about 21,000 hours, while this time for an alliance of Iranian airlines was about 19,516 hours. In fact, when we use the alliance of Iranian airlines and their under-controlled hubs, travel time per traveler was decreased by about 59 minutes on average.

\section{3. Sensitivity Analysis and Managerial Insights}

To assess the impact of quality and safety of follower airline and the number of their under-controlled hubs in value of $z$, We implemented the model with different values for $U_{f}, U_{l}, S_{f}, S_{l}$ and $|K|$ in the mode of uncertainty. The results are presented in Tables 3 to 4. Table 3 indicates the impact of the number of airports controlled by follower airline on the value of the objective function.

TABLE 2. Discount factor

\begin{tabular}{lccc}
\hline Discount factor & $\begin{array}{c}\boldsymbol{\beta}_{\mathbf{1}}=\mathbf{1}, \\
\boldsymbol{\gamma}_{\mathbf{1}}=\mathbf{0 . 9 0 4}\end{array}$ & $\begin{array}{c}\boldsymbol{\beta}_{\mathbf{1}}=\mathbf{0 . 9}, \\
\boldsymbol{\gamma}_{\mathbf{1}}=\mathbf{1}\end{array}$ & $\begin{array}{c}\boldsymbol{\beta}_{\mathbf{1}}=\mathbf{0 . 9}, \\
\boldsymbol{\gamma}_{\mathbf{1}}=\mathbf{0 . 9 6}\end{array}$ \\
\hline$z$ & 829,276 & 850,640 & 829,358 \\
\hline
\end{tabular}

TABLE 3. Sensitivity analysis of the number of airports

\begin{tabular}{ccccc}
\hline$|\boldsymbol{K}|$ & $\mathbf{5}$ & $\mathbf{6}$ & $\mathbf{7}$ & $\mathbf{6}$ \\
\hline$z$ & 779,997 & 806,761 & 812,335 & 822,896 \\
\hline
\end{tabular}

TABLE 4. Sensitivity analysis of quality and safety

\begin{tabular}{llll}
\hline$\frac{u_{f}}{U_{l}}, \frac{s_{f}}{s_{l}}$ & $\mathbf{0 . 5 , 0 . 2}$ & $\mathbf{0 . 4 , 0 . 3}$ & $\mathbf{0 . 5 , 0 . 3}$ \\
\hline$z$ & $1,028,620$ & $1,234,344$ & $1,542,930$ \\
\hline
\end{tabular}

As shown in Table 3, an increase in the number of hub airports of the follower airline led to an increase in the value of the objective function. By simple calculations, it could be shown that adding one airport to airports controlled by follower airline led to a $1 \%$ increase in revenue of follower airline.

Table 4 shows the impact of the quality and safety of the follower airline on the value of the objective function. The first column of Table 4 showed that a $10 \%$ increase in quality of follower airline led to a $25 \%$ increase in the value of the objective function. While a $10 \%$ increase in safety of follower airline (second column of Table 4) led to a $50 \%$ increase in revenue of follower airline. Therefore, it could be concluded that the safety of airlines was more effective in traveler attraction and revenue increase. The third column of Table 4 showed that a $10 \%$ increase in each of the parameters of quality and safety of follower airline led to a nearly $88 \%$ increase in the value of the objective function.

To examine the impact of the number of uncertain parameters, we considered Table 5. According to Table 5 , the more uncertain parameters in the model, the worst value of the objective function. For example, when all 25 parameters $w_{i j}$ were uncertain, with the following deviation value:

$$
\hat{w}_{i j}=0.3 * w_{i j}
$$

TABLE 5. Impact of uncertain parameter

\begin{tabular}{llc}
\hline $\boldsymbol{\Gamma}_{\boldsymbol{i j}}^{\text {const }}$ & $\hat{\boldsymbol{w}}_{i j}$ & $\mathbf{z}^{*}$ \\
\hline \multirow{3}{*}{15} & $0.1\left(w_{i j}\right)$ & 752,522 \\
& $0.2\left(w_{i j}\right)$ & 703,113 \\
& $0.3\left(w_{i j}\right)$ & 648,804 \\
\hline \multirow{2}{*}{20} & $0.1\left(w_{i j}\right)$ & 743,839 \\
& $0.2\left(w_{i j}\right)$ & 685,724 \\
& $0.3\left(w_{i j}\right)$ & 622,722 \\
\hline \multirow{2}{*}{25} & $0.1\left(w_{i j}\right)$ & 725,891 \\
& $0.2\left(w_{i j}\right)$ & 649,382 \\
& $0.3\left(w_{i j}\right)$ & 568,208 \\
\hline
\end{tabular}


Then, the value of the objective function was equal to $\$ 568,208$, which was $\$ 254,688$ lower than the optimal value of a certain model $(\$ 822,896)$. Some managerial insights from the study findings are summarized below.

However, calculations results based on cost and travel time parameters, suggest the transfer of some international travelers through Iranian airports, but given the low quality of services provided at these airports, it is necessary for satisfying the travelers and increasing numbers of them in the future, increase the quality of services provided in them. Given the geographical location of airports in Iran, under the conditions of postsanctions and if the quality and satisfaction of Iranian airlines reach the level of quality and satisfaction of Emirate airline, their earnings will increase to 2.5 times the current level.

\section{CONCLUSION}

In this paper, to determine the exact location of hubs we develop the model of Marianov et al. [1] by defining a new type of capture sets under uncertainty in which the follower airline attracted a percentage of the leader airline's travelers. We divided the nominated airports for follower airline's hub into six independent categories by comparing parameters of travel time, travel cost, and service quality of airports of follower airline and leader airline. By employing multivariate Lagrange interpolation function, we determined the degree of importance of time and cost of travel from the traveler's perspective. By using the degree of importance of time and cost of travel, as well as comparing the parameters of quality, safety, and delays between leader airline and follower airline, we allocated travelers to those six-fold sets. Based on the seasonal demand of travelers used by local travelers; we considered travel demand of hubs as uncertain parameters. To determine the robust counterpart of this category of hub location problem, a robust optimization method of Bertsimas and Sim [5] was used. The results show that the follower airline's income has a considerable growth compared to the Marianov et al. [1] model and can absorb nearly $2 \%$ of travelers of the leader airline due to lower travel costs and travel time compared to that of leader airline. By examining the change in model's parameters, we found that improvement of quality and safety of constituent airlines in follower airlines and an increase in the number of their under-controlled hubs had a positive impact on revenue of follower airline.

\section{REFERENCES}

1. Marianov, V. Serra, D. and ReVelle, C., "Location of hubs in a competitive environment", European Journal of Operational Research, Vol. 114 , No. 2, (1999), 363-371.
2. Wagner, B., "A note on "location of hubs in a competitive environment"", European Journal of Operational Research, Vol. 184, No. 1, (2008), 57-62.

3. Soyster, A. L., "Convex programming with set-inclusive constraints and applications to inexact linear programming", Operations research, Vol. 21, No. 5, (1973), 1154-1157.

4. Ben-Tal, A. and Nemirovski, A., "Robust solutions of uncertain linear programs", Operations research letters, Vol. 25, No.1, (1999), 1-13.

5. Bertsimas, D. and Sim, M., "The price of robustness", Operations research, Vol. 52, No.1, (2004), 35-53.

6. Sasaki, M. and Fukushima, M., "Stackelberg hub location problem", Journal of the Operations Research Society of Japan, Vol. 44, No. 4, (2001), 390-402.

7. Sasaki, M., "Hub network design model in a competitive environment with flow threshold", Journal of the Operations Research Society of Japan, Vol. 48, No. 2, (2005), 158-171.

8. Sasaki, M. Campbell, J. F. Krishnamoorthy, M. and Ernst, A. T., "Designing hub and spoke transportation systems for a competitive environment", in International Conference on Industrial Engineering and Systems Management, (2009), 13-15.

9. Eiselt, H. A. and Marianov, V., "A conditional p-hub location problem with attraction functions", Computers \& Operations Research, Vol. 36, No. 12, (2009), 3128-3135.

10. Lin, M. H., "Strategic airline alliances and endogenous Stackelberg equilibria", Transportation Research Part E: Logistics and Transportation Review, Vol. 40, No. 5, (2004), 357-384.

11. Zou, L. and Chen, X., "The effect of code-sharing alliances on airline profitability", Journal of Air Transport Management, Vol. 58, (2017), 50-57.

12. Yimga, J. O., "Airline code-sharing and its effects on on-time performance", Journal of Air Transport Management, Vol. 58, (2017), 76-90.

13. Nikoofal, M. E. and Sadjadi, S. J., "A robust optimization model for p-median problem with uncertain edge lengths", The International Journal of Advanced Manufacturing Technology, Vol. 50, No. 1-4, (2010), 391-397.

14. Ghaffari-Nasab, N. Ghazanfari, M. and Teimoury, E., "Robust optimization approach to the design of hub-and-spoke networks", The International Journal of Advanced Manufacturing Technology, Vol. 76, No. 5-8, (2015), 1091-1110.

15. Zetina, C. A. Contreras, I. Cordeau, J. F. and Nikbakhsh, E., "Robust uncapacitated hub location", Transportation Research Part B: Methodological, Vol. 106, (2017), 393-410.

16. Tavakkoli-Moghaddam, R. Gholipour-Kananib, Y. and Shahramifarc, M., "A multi-objective imperialist competitive algorithm for a capacitated single-allocation hub location problem", International Journal of Engineering-Transactions C: Aspects, Vol. 26, No. 6, (2013), 605-612.

17. Ghodratnama, A., Tavakkoli-Moghaddam, R. and Baboli, A., "Comparing three proposed meta-heuristics to solve a new p-hub location-allocation problem", International Journal of Engineering-Transactions C: Aspects, Vol. 26, No. 9, (2013), 1043-1058.

18. Bashiri, M. and Rezanezhad, M., "A reliable multi-objective phub covering location problem considering of hubs capabilities", International Journal of Engineering-Transactions B: Applications, Vol. 28, No. 5, (2015), 717-729.

19. Alizadeh, Y. Tavakkoli-Moghadam, R. and Ebrahimnejad, S., "A new multi-objective model for a capacitated hub covering problem solving by two multi-objective evolutionary algorithms", International Journal of Mathematics in Operational Research, Vol. 9, No. 1, (2016), 99-124. 
20. Karimi, H., Bashiri, M. and Nickel, S., "Capacitated single allocation p-hub covering problem in multi-modal network using tabu search", International Journal of EngineeringTransactions C: Aspects, Vol. 29, No. 6, (2016), 797-808.

21. Saniee, K., "A Simple Expression for Multivariate Lagrange Interpolation", SIAM undergraduate research online, Vol. 1, No. 1, (2008), 1-9.

\section{APPENDIX A}

Data Needed for Implementation of the Model Origins, destinations, and hub candidates of the follower airline are presented in Tables A1 and A2. Also, the service quality of airports and the capacity of hub candidates as well as the minimum number of passing travelers for being them as the hub are presented in Table A3. The weekly average number of travelers transferred by Emirate airport in the first six months of the year 2018 is presented in Table A4.

TABLE A1. Origins and destinations

\begin{tabular}{ccccc}
\hline \multicolumn{2}{c}{ Origin } & & \multicolumn{2}{c}{ Destination } \\
\cline { 1 - 2 } \cline { 5 - 5 } Label & Airport & & Label & Airport \\
\hline$i_{1}$ & Hamburg & & $j_{1}$ & Beijing \\
$i_{2}$ & London & & $j_{2}$ & Bangkok \\
$i_{3}$ & Stockholm & & $j_{3}$ & Kuala Lumpur \\
$i_{4}$ & Paris & & $j_{4}$ & Delhi \\
$i_{5}$ & Rome & & $j_{5}$ & Karachi \\
\hline
\end{tabular}

TABLE A2. Hub candidates of follower airline

\begin{tabular}{lrrrrrrrr}
\hline Label & $k_{1}$ & $k_{2}$ & $k_{3}$ & $k_{4}$ & $k_{5}$ & $k_{6}$ & $k_{;}$ & $k_{8}$ \\
\hline Airport & $\begin{array}{l}\text { Imam } \\
\text { Khomeini }\end{array}$ & Mashhad & Tabriz Isfahan Shiraz & $\begin{array}{c}\text { Bandar } \\
\text { Abbas }\end{array}$ \\
\hline
\end{tabular}

TABLE A3. Service quality, capacity and Minimum number of passing travelers for being airport, as a hub

\begin{tabular}{lccc}
\hline Airport & $\begin{array}{c}\text { Service } \\
\text { quality }(*)\end{array}$ & Capacity & $\begin{array}{c}\text { Minimum } \\
\text { number }\end{array}$ \\
\hline$k_{1}$ & 3 & 125,136 & 2,700 \\
$k_{2}$ & 3 & 110,469 & 2,400 \\
$k_{3}$ & 3 & 33,721 & 700 \\
$k_{4}$ & 3 & 57,813 & 1,300 \\
$k_{5}$ & 3 & 61,018 & 1,400 \\
$k_{6}$ & 1 & 34,009 & 700 \\
$k_{7}$ & 1 & 21,111 & 500 \\
$k_{8}$ & 1 & 18,989 & 400 \\
Dubai & 4 & - & - \\
\hline
\end{tabular}

TABLE A4. Number of travelers

\begin{tabular}{lrrrrc}
\hline Number of traveler & $\boldsymbol{j}_{\mathbf{1}}$ & $\boldsymbol{j}_{\mathbf{2}}$ & $\boldsymbol{j}_{\mathbf{3}}$ & $\boldsymbol{j}_{\mathbf{4}}$ & $\boldsymbol{j}_{\mathbf{5}}$ \\
\hline$i_{1}$ & 4,020 & 3,350 & 1,340 & 4,020 & 4,020 \\
$i_{2}$ & 4,020 & 2,680 & 1,340 & 4,020 & 3,350 \\
$i_{3}$ & 4,020 & 4,020 & 2,010 & 4,020 & 3,350 \\
$i_{4}$ & 2,010 & 2,010 & 670 & 2,010 & 2,010 \\
$i_{5}$ & 4,020 & 4,020 & 1,340 & 4,020 & 4,020 \\
\hline
\end{tabular}

\section{Development of a Model for Locating Hubs in a Competitive Environment under Uncertainty: A Robust Optimization Approach}

F. Nourzadeha, S. Ebrahimnejad ${ }^{b}$, K. Khalili-Damghania, A. Hafezalkotob ${ }^{a}$

a Department of Industrial Engineering, South Tehran Branch, Islamic Azad University, Tehran, Iran

${ }^{b}$ Department of Industrial Engineering, Karaj Branch, Islamic Azad University, Karaj, Iran

PAPER INFO

\section{Paper history:}

Received 25 August 2019

Received in revised form 30 September 2019

Accepted 08 November 2019

\section{Keywords:}

Competitive Location

Multivariate Lagrange Interpolation

Function

Location $\mathrm{Hub}$

Robust Optimization

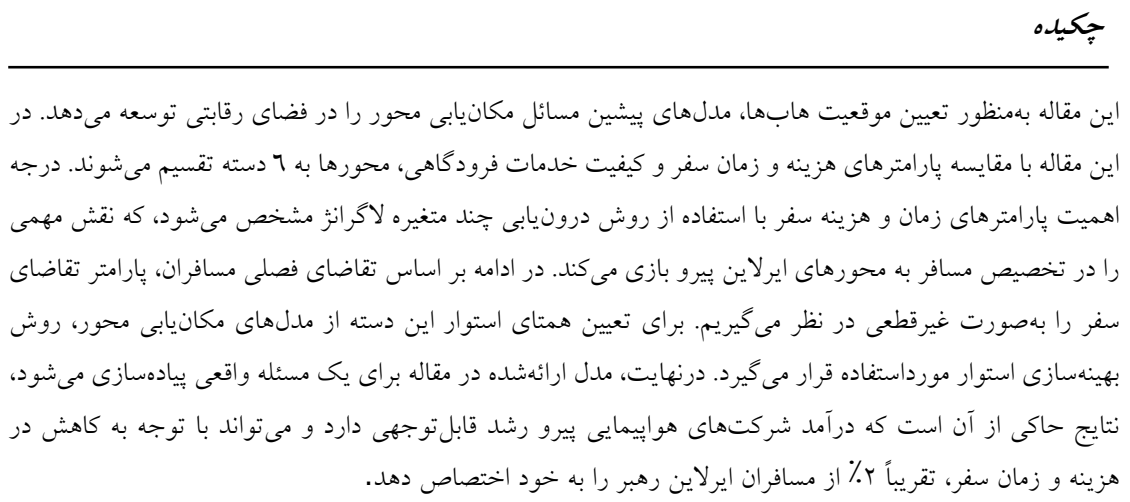

doi: 10.5829/ije.2020.33.01a.14 